\title{
Fetal maternal immune cross talking and vaccination during pregnancy
}

\section{Panagiota Masoura', Georgia Koutsogeorgopoulou' ${ }^{1}$, Diamanto Tsianni' Anastasia Kourtesa ${ }^{1}$, Kalliopi Pappa ${ }^{2}$}

'National and Kapodistrian, University of Athens, School of Medicine

${ }^{2}$ First Department of Obstetrics and Gynecology, Alexandra Hospital, National and Kapodistrian, University of Athens, School of Medicine

\section{Corresponding Author}

Kalliopi Pappa, $1^{\text {st }}$ Department of Obstetrics and Gynecology, Alexandra Hospital, National and Kapodistrian University of Athens, Greece

\section{Abstract}

Maternal adaptations in pregnancy induce complex physiological changes of the immune system which protect mother's health and ensure the accommodation of the growing embryo. The innate immunity is amplified and the adaptive immunity is partially suppressed, preserving the ability to produce antibodies. Vaccination during pregnancy constitutes a fundamental preventive measure in the antenatal care, as it achieves both the immunization of the mother and the baby. Besides passive immunization through the placenta, also active transfer of IgG via placenta occurs throughout pregnancy. Live attenuated vaccines (LAV) are contraindicated in pregnant women, while the others should be recommended if benefits overshadow risks.

Key words: Pregnancy, Immunization, Placenta, Antibodies, Vaccines, Perinatal outcome

\section{Introduction}

Pregnancy has been considered as a period of immunosuppression for the mother ${ }^{1,2}$. Nowadays, these data seems to be overturned, since immune system adaptations keep maternal defense ability in satisfactory levels ${ }^{2}$. Moreover these changes maintain an immunologic tolerance to the hemi-allogenic embryo which partially consists of paternal antigens ${ }^{3}$. Immunological changes take place to almost all the immune cell lines. Regarding innate immunity and especially the antigen presenting cells (APCs), the adaptations include:

- The rise in number and effectiveness of phagocytes and granulocytes ${ }^{2}$. This is strongly supported by the rise of granulocyte and macrophage colony stimulating factors (G-CSF, M-CSF) in maternal serum $^{2}$

- The rise in a-defensins which displaying either direct antimicrobial activity and/or immune sig- 
naling activities ${ }^{2}$ and

- The rise in dendritic cells, especially in plasmatocytoid dendritic cells ${ }^{2}$.

Regarding adaptive immunity it seems to incur different changes, including:

- The reduction of the natural killer (NK) cells and CD4, especially after the $20^{\text {th }}$ week of gestation ${ }^{2}$ and the reduction of T-lymphocytes as pregnancy progresses, particularly after the $2^{\text {nd }}$ trimester ${ }^{2}$.

- Serum cytokines also diminished following the pattern of T-lymphocytes. The Th1 cytokines reduction appears to be more profound than the Th2 cytokines ${ }^{2,4}$.

- B-lymphocytes decrease during pregnancy, especially in the $3^{\text {rd }}$ trimester ${ }^{2}$.

The trigger factors of these changes are still obscure. However, the hormonal changes during pregnancy may play a central role as they are involved in a number of body functions, including the immune system regulation. Estrogens, for example, are known to suppress the production of B-lymphocytes in the bone marrow ${ }^{4}$. On the other hand, estrogens seem to help B-lymphocytes to survive for longer periods in circulation and also robust their ability to produce antibodies ${ }^{4}$. Interestingly, the reduction of B-lymphocytes in mother's serum is not accompanied with an equal maternal antibody reduction or weaker immune response ${ }^{2}$.

Pregnancy adaptations affect maternal response to infections and vaccination. Studies on maternal vaccination, have shown that these adaptations do not suppress antibody production throughout pregnancy and especially during the $3^{\text {rd }}$ trimester were most vaccinations are performed if necessary ${ }^{5,6}$. Influenza vaccines studies have shown that the percentage of response differs during the course of pregnancy with the most weak to be noted before the $13^{\text {th }}$ week and the most robust after the $34^{\text {th }}$ gestational week ${ }^{5}$. Other studies have shown decreased immunological responce $^{7}$ or a result of memory response from the already sensitized mature maternal B-lymphocytes (boosting effect) ${ }^{5,8}$.

Regarding the safety of vaccination during pregnancy, the majority of the studies show that they are safe and effective not only for the mother herself, but also for the fetus and newborn ${ }^{6,9,10}$. In a small study, there was a correlation between first trimester vaccination and the delivery of small for gestational age (SGA) newborns ${ }^{11}$. Interestingly, one study showed a correlation between strong immune response of the pregnant woman and preterm birth ${ }^{11}$. Women that delivered preterm after being vaccinated against H1N1, had higher percentages of seroconversion versus women who delivered full term ${ }^{11}$.

\section{The impact of vaccines in fetus}

The highest transport of maternal antibodies to the fetus happens during the last 4 weeks of the third trimester. This replica of maternal chemical immunity provides equal protection to infants during the first 6 months of life. Therefore, this mechanism is the basic modulator of neonatal immunity ${ }^{12,18}$.

Detection of maternal IgG in the umbilical cord blood is observed as early as the 8th to 10 th week. In the second trimester, during the 17 th to 22 nd week almost $10 \%$ of maternal IgG is transferred to the fetus while up to $50 \%$ of maternal IgG is transferred from the 28th to 32nd week. The greatest quantity of IgG is transferred after the 32nd week from the extended surface of the developing placenta and after the 36th week of pregnancy an abrupt increase of IgG in the umbilical cord blood is detected. The levels of IgG in the fetal blood often exceed the $20-30 \%$ of maternal levels in the 37 th to 40 th week of pregnancy ${ }^{13,17}$. (Figure 1). After maternal vaccination the increasing in infant IgG titers occurs in 2 weeks ${ }^{18}$.

\section{Transportation of maternal antibodies}

IgG antibodies are the only class of maternal anti- 


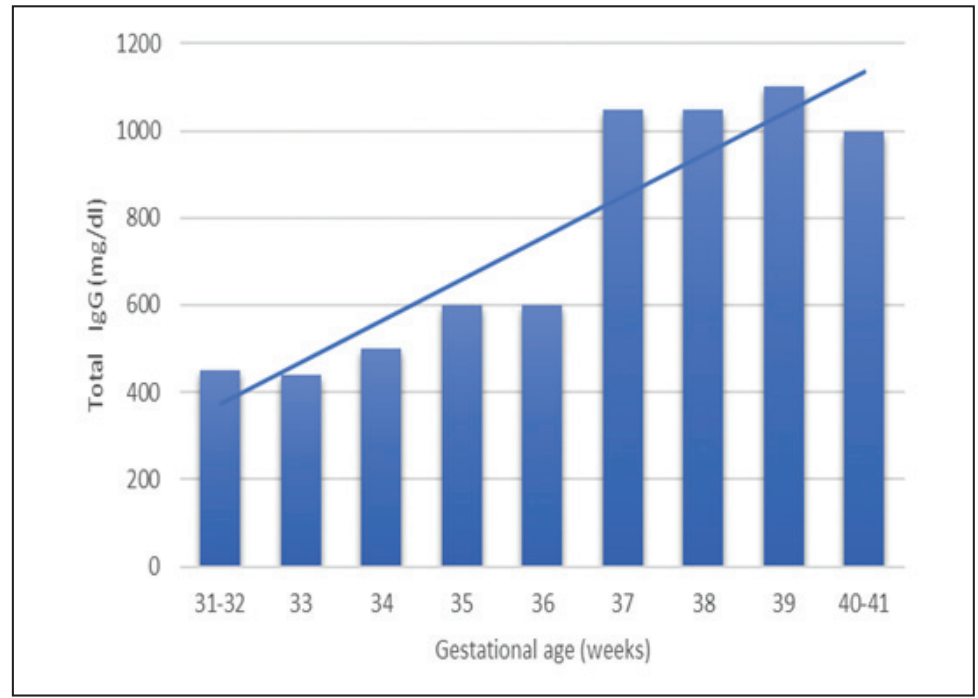

Figure 1. The bars depict the total concentration of IgG antibodies in mg/dl per gestational week.

bodies that crosses extensively the human placenta. The IgG are transported either as a single molecule or as an IgG-immune complex (IgG-IC) ${ }^{12,13}$.

The neonatal FcRn receptor binds the Fc portion of IgGs at acidic $\mathrm{pH}(\leq 6.5)$ and mediates IgG trafficking. FcRn is expressed in placental cytotrophoblast, in endothelium, in epithelium, macrophages (Mfs), and dendritic cells (DCs), in hematopoietic cells and in the mucinous cells of the fetal lung and gastrointestinal tract ${ }^{12,13,14,19}$.

IgG enter in cells from the micro-environment (fluid or blood) through phagocytosis. In an acidic compartment ( $\mathrm{pH} \leq 6.5$ ) FcRn binds to IgG and recycles it by accompanied it back to the cell surface where releases it (pH 7.0-7.4). (Figure 2) FcRn binds preferentially with IgG and in this way, a high distribution and levels of IgG all over the body is ensured. FcRn receptor expression depends on gestational age and it is over-expressed in the third trimester of pregnancy, leading to a higher rate of IgG transportation during late pregnancy, as said above $\mathrm{e}^{13,19}$.

The placental transport system is extremely selective, as it allows only IgG antibodies to pass through and blocks the transport of other major immunoglobulin classes including $\operatorname{IgE}, \operatorname{IgM}, \operatorname{IgA}^{15}$. Maternal IgG antibodies are divided into 4 subclasses (IgG1, IgG2, IgG3 and IgG4). Every subclass response to vaccines is varied up on the vaccine components. Vaccines containing protein antigens (e.g. tetanus toxoid) induce the production of IgG1 antibodies, whereas vaccines containing polysaccharide antigens (e.g. pneumococcus) induce the production of IgG2 antibodies. IgG1 and IgG4 subclasses own a higher affinity for the FcRn receptor compared to IgG2 and IgG3. For that reason IgGs exhibit different transportation ability with the IgG1 presenting the highest preferential transport ability while the IgG2 the lowest. The IgG transport shows saturation kinetics, because it is paused when IgG levels reach up to $15 \mathrm{~g} / \mathrm{L}$. Thus high levels of IgG antibodies lead to the saturation of FcRn receptors and consequently to an unbound IgG fraction that it will be degraded by lysosomal enzymes within the vesicles ${ }^{12,13,17}$.

Factors that positively affect placental transmission are late gestational age, current maternal vac- 


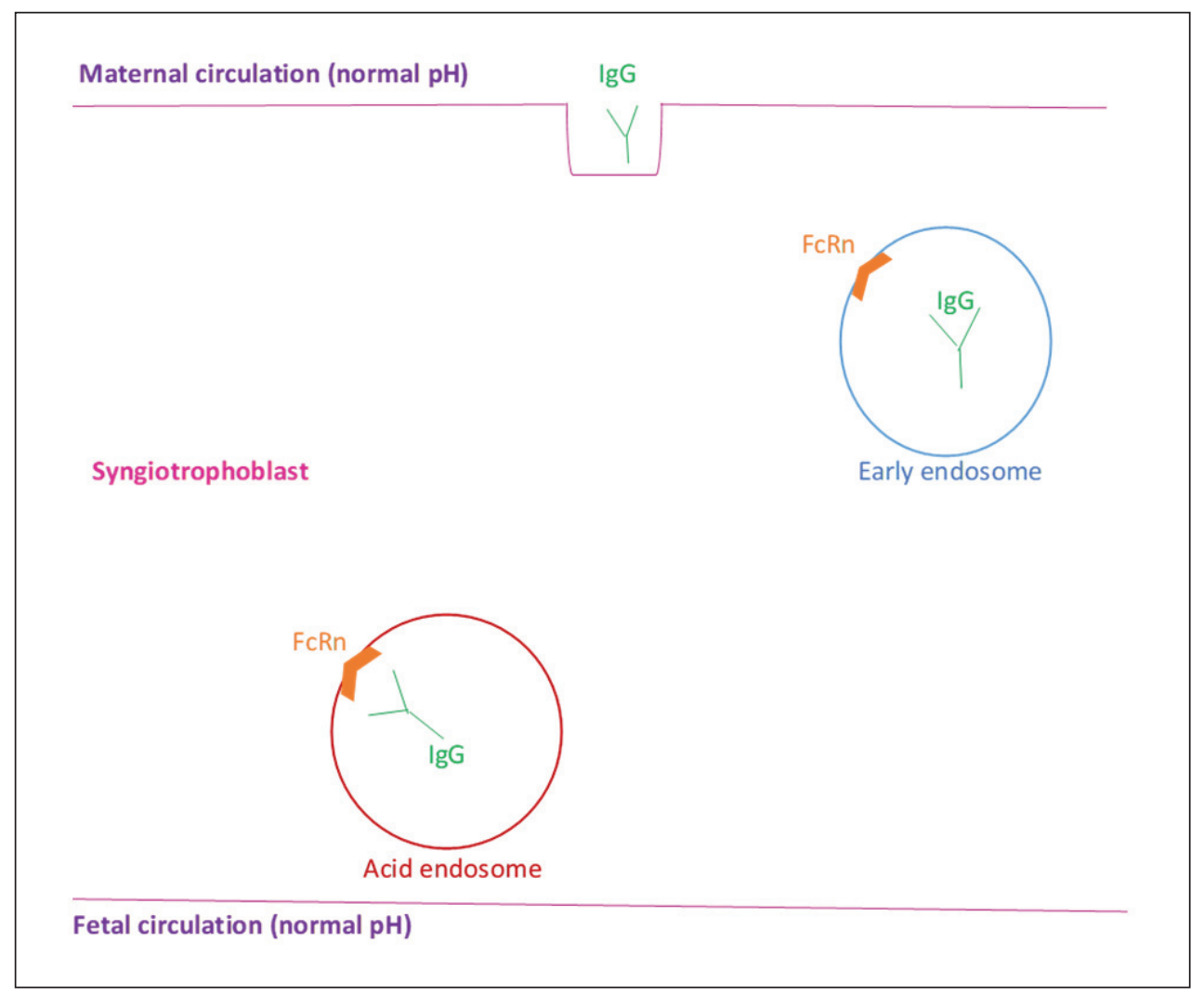

Figure 2. Syncytotrophoblast cells, which are soaked by maternal blood, endocytes maternal IgG, into endosomes. The FcRn receptor is expressed on the inner surfaces of the endosomes. Two IgG molecules bind to each FcRn receptor under acidic environment and get protected from degradation by lysosomal enzymes. The acidic endosomes are transported and fused to the membrane on the fetal compartment of the syncytotrophoblast, where the pH is normal and IgG is released from the FcRn receptor into the fetal circulation and subsequently can be recycled performing another transport cycle. The FcRn receptor shows much higher affinity for IgG in acidic environments $(\mathrm{pH} 6)$ compared to the normal (pH 7.4) which characterizes maternal and fetal blood.

cination, balanced maternal nutrition and the male gender ${ }^{12}$ while transmission barriers are developed in placenta disorders (insufficiency), maternal immunity diseases (HIV, malaria) and maternal hypergammaglobulinemia ${ }^{13,17,18}$.

In the fetal circulation maternal IgG bind to FcRn receptors expressed at barrier sites, such as the blood-brain barrier, the glomerular filter, and the intestinal epithelium and are transported to promote fetal defense or control ${ }^{13,19}$. FcRn receptors on the intestinal epithelial cells bind IgG molecules from the ingested amniotic fluid mediating their intracellular transport to the fetus and promoting the activation and maturation of the fetal immune system in the first months of pregnancy ${ }^{14,16,19}$.

\section{Vaccines contraindicated in pregnancy}

Vaccines containing live attenuated microorganisms are contraindicated in pregnancy as they reach the fetal circulation through the placenta and consequently have the potential to cause infection and congenital anomalies to the fetus ${ }^{20}$. 
Therefore, the following vaccines should not be given to pregnant women ${ }^{20}$ :

1. MMR (measles, rubella, mumps)

2. Varicella

3. Zoster

4. Human Papillomavirus (HPV)

5. BCG

\section{MMR vaccine}

- It contains live attenuated measles, rubella and mumps viruses and therefore should not be used during pregnancy as there is a risk of maternal death and fetal infection or teratogenicity ${ }^{20}$

- It is suggested avoiding conception for 4 weeks after vaccination $^{21}$

- Routine screening of childbearing potential women for possible pregnancy before vaccination is not recommended ${ }^{22}$

- All pregnant women should be tested for immunity to rubella and those without immunity should be vaccinated immediately after delivery ${ }^{21}$

- In unvaccinated women when exposed to the viruses during pregnancy, IgG immunoglobin should be administrated within 6 days $^{23}$

\section{Varicella vaccine}

- It contains live attenuated varicella viruses ${ }^{20}$

- It is recommended to avoid conception for 1 month after vaccination ${ }^{20}$

- Routine screening of childbearing potential women for possible pregnancy before the vaccination is not recommended ${ }^{22}$

- Routine screening for varicella should be done in early pregnancy in all pregnant women ${ }^{21}$

- On confirmed exposure to the virus, a varicellazoster immunoglobulin should be administered immediately ${ }^{21}$

- Pregnant women infected with the virus should take two doses of the vaccine after delivery, the first immediately after delivery and the second after 4-8 weeks ${ }^{21}$

\section{Herpes Zoster vaccine}

- Two types of vaccine are available: a recombinant zoster vaccine (RZV) and a live zoster vaccine $(\mathrm{ZVL})^{20}$

- The RZV has not been studied in pregnant women ${ }^{20}$

- The CDC suggests that RZV vaccination should be given after pregnancy ${ }^{20}$

- Pregnancy should be avoided for 3 months after vaccination $^{22}$

\section{BCG}

- Regarding BCG vaccination, no adverse effects have been documented concerning the fetus but the data supporting the safety of BCG vaccine are inadequate and therefore more studies are required ${ }^{20}$.

\section{Human Papillomavirus vaccine (HPV)}

- Three types of HPV vaccines are available (2-valent, 4-valent and 9-valent). They are all recombinant vaccines $^{20}$

-When vaccination is applied soon after conception, then we defer the other two doses after delivery ${ }^{20}$

- Routine screening of childbearing potential women for possible pregnancy before the vaccination is not recommended ${ }^{22}$

- The vaccine has not been associated with adverse pregnancy outcomes or spontaneous abortion, late fetal death and congenital anomalies ${ }^{20}$

\section{Vaccines which should be used only under certain circumstances}

The following vaccines should be given only when the benefits outweigh the risks ${ }^{20}$ :

1. Hepatitis A

2. Hepatitis B 
3. Pneumococcal

4. Meningococcal

5. Polio (IPV)

6. Typhoid fever

7. Smallpox

\section{Hepatitis A vaccine}

- Hepatitis A vaccine contains dead inactivated hepatitis A viruses ${ }^{20}$

- The CDC recommends the use of vaccine in pregnant women at high risk of exposure ${ }^{20}$ :

- Intravenous drug users

- Travellers to countries with poor sanitation (sub-Saharan Africa and South Asia)

- Women in close contact with HAV-infected people or if they have an HAV-infected sexual partner

- There are insufficient data about safety during pregnancy. However, the vaccine is rare to cause harm to the fetus ${ }^{21}$ and a small number of studies agree that there are no adverse effects in pregnant women or their infants after hepatitis A vaccination during pregnancy ${ }^{20}$

- In addition, the damage of hepatitis A infection during pregnancy is greater than the danger from vaccination. Premature birth, fetal ascites, placental abruption, vaginal bleeding or premature rupture of membranes are some of the adverse outcomes after hepatitis A infection. ${ }^{20}$

\section{Hepatitis B vaccine}

- It is a recombinant vaccine containing the hepatitis $B$ virus surface antigen (HBsAg) ${ }^{20}$

- Data on vaccine safety are limited and are not associated with adverse maternal or fetal outcomes ${ }^{21}$

- However, it is only recommended for completing the vaccination schedule started before conception and for pregnant women who have not been vaccinated before and are at high risk for infection (multiple sexual partners in the last 6 months, HBsAg positive partner, HIV positive, intravenous drug users or those who had previous STDs) ${ }^{20}$

- Routine screening (HBsAg) for all pregnant women should be recommended ${ }^{21}$

- Pregnant women positive for HBsAg, should be tested for HBV DNA and if a high viral load is diagnosed then antiviral therapy should be reccomented. ${ }^{21}$

- Intrauterine transmission is most likely to happen during mother's primary infection towards to the end of pregnancy ${ }^{21}$ In this case, the newborn is given the immunoglobulin and the hepatitis $B$ vaccine within 12 hours of birth ${ }^{21}$

\section{Pneumococcal vaccine}

- There are two unconjugated polysaccharide vaccines PCV13 and PPSV23

- There are currently insufficient data to support routine PCV13 (conjugated polysaccharide) or PPSV23 (unconjugated polysaccharide) during pregnancy $^{21}$

- In a study of PPSV23 effects on pregnancy, it did not show an increase in spontaneous abortion, teratogenesis, preterm birth or congenital anomalies $^{21}$

- PPSV23 vaccine should be administered during pregnancy only in women in high risk for ${ }^{20}$ :

- High risk of pneumococcal infection ${ }^{20}$

- Chronic diseases (congestive heart failure, cardiomyopathy, chronic lung disease, cirrhosis, diabetes)

- Immunosuppression (congenital immunodeficiency, HIV infection, hematological malignancies, bone marrow or organ transplantation, treatment with alkylating agents and antimetabolites or corticosteroids)

- Chronic renal failure and nephrotic syndrome

- Ear cochlear implants

- Vaccine safety is proven for the second and third trimester while there are few data for the first trimester ${ }^{20}$ 


\section{Meningococcal vaccine}

- Two types of meningitis vaccine are available: the group $\mathrm{A}, \mathrm{C}, \mathrm{W}-135$ and $\mathrm{Y}$ vaccine (MenACWY) and the meningococcal group $B$ vaccine $(\mathrm{MenB})^{20}$

- MenACWY vaccination is not contraindicated during pregnancy based on the CDC recommendations. ${ }^{20}$ Vaccine seems to be safe and studies have associate vaccination mostly with spontaneous abortion $^{20,21}$

- MenB is contraindicated for pregnant women. However vaccination is recommended when the benefits outweigh the potential risks ${ }^{20}$ such as

- Anatomical or functionall asplenia

- Complement components deficiency

- Travellers to endemic areas (sub-Saharan Africa)

- Microbiologists frequently exposed to Neisseria meningitides

\section{Poliomyelitis vaccine (IPV)}

- There are two types of polio vaccines: one with inactivated viruses (Salk or IPV vaccine) and one with live attenuated viruses (Sabin or OPV vaccine) which is currently not licensed globally due to the vaccine-associated paralytic polio (VAPP) ${ }^{20}$ Although no major adverse effects have been reported after IPV administration, vaccination of pregnant women should be avoided ${ }^{20}$

However, the CDC recommends IPV for pregnant women at high risk of infection. High risk groups include travellers to endemic countries ${ }^{20}$

\section{Typhoid fever vaccine}

- The two types of typhoid fever vaccine are the live attenuated vaccine and the Vi polysaccharide which contain the Vi capsular antigen ${ }^{21}$

- The attenuated vaccine is contraindicated ${ }^{22}$ during pregnancy and if it is necessary to vaccinate a pregnant woman (high risk group), then the $\mathrm{Vi}$ polysaccharide one should be used ${ }^{22}$

- Vaccination during pregnancy is recommended in high risk groups such as:

- travellers to countries with poor sanitation or endemic areas

- persons in close contact with typhoid-infected people

- workers exposed to Salmonella Typhi ${ }^{20}$

It is recommended to delay the vaccination until the second or third trimester to avoid possible teratogenic effects ${ }^{20}$

\section{Smallpox vaccine}

- The vaccine contains live attenuated strains of variola ${ }^{20}$ Due to the severe consequences after fetal infection (fetal or neonatal death ${ }^{1}$ ) the smallpox vaccine is contraindicated for women who are pregnant or might be pregnant within 4 weeks after vaccination ${ }^{22}$ although there are no clear and consistent data of adverse perinatal outcome after vaccination.

- However, pregnant women who had been directly exposed to the virus should be vaccinated as the risks to the mother and fetus are greater than the potential risks of vaccination ${ }^{22}$

\section{Consulting a woman after a contraindicated vaccination during pregnancy}

Vaccines containing live attenuated pathogens, such as MMR, BCG, Varicella, smallpox e.t.c., are contraindicated during pregnancy as they present a hypothetical risk of overcoming the placental barrier and infect the fetus ${ }^{20}$.

The reports for poor perinatal outcome are weak, due to the lack of studies. If the vaccine is inadvertently administered during pregnancy or when pregnancy happens earlier than 28 days after vaccination, consultation should be done about the potential fetal risks $^{26}$. Regarding rubella, the Advisory Committee on Immunization Practices (ACIP) gathered data from vaccination registries in the Europe (years 1971-1990 and 2001-2004) and the USA (1971-1988). Among 
680 live births to rubella-susceptible women, none of the infants was diagnosed with Congenital Rubella Syndrome (CRS) ${ }^{25}$. Studies in Canada, Latin America and Islamic Republic of Iran reveal that among 3500 rubella-susceptible women, who had been vaccinated against rubella shortly before or during pregnancy, there was none case presented congenital defects (e.g. hearing loss, cataract, mental retardation, congenital heart disorders) ${ }^{25}$. According to the available data, vaccination during or shortly before pregnancy should not be a reason of pregnancy termination ${ }^{20}$.

In 1995 a study was conducted by the Merk and Co. in collaboration with the CDC, in order to examine the maternal and fetal outcomes, after the inadvertent vaccination of women with the varicella vaccine within 3 months before pregnancy period or during pregnancy ${ }^{27}$. None case of congenital varicella syndrome (CVS) or birth defects documented (e.g limb hypoplasia, microcephaly, low birth weight, cortical atrophy, chorioretinitis, cataract) ${ }^{20}$. The rate of major birth defects was similar to the background population $(3.2 \%)^{20}$. The study results do not exclude the contingent risk to the fetus, however the risk is low and pregnancy termination is irrational ${ }^{20}$.

According to a review article of 2014, about the safety of vaccines during pregnancy, it seems that live attenuated vaccines (e.g. measles, mumps, rubella) do not rise safety concern for the fetus and the infant ${ }^{25}$. The benefits of vaccination outweigh the potential risks and the results are reassuring that the risk is absent or minimum ${ }^{25}$.

For the recombinant technology HPV vaccine, and especially for the quadrivalent HPV vaccine (qHPV) more data are available in the literature comparing to the prior ones. The rate of adverse pregnancy outcomes (e.g. spontaneous abortion, congenital anomalies, late fetal death) is not raised by the vaccination with the qHPV ${ }^{28}$. The data for the 9-valent HPV vaccine are limited, but equally reassuring. The results from a combined analysis study of seven phase-III clinical trials on the 9-valent HPV vaccine, have shown that the spontaneous abortion frequency was higher in the inadvertent vaccination of pregnant women with the $9 \mathrm{v}-\mathrm{HPV}$ vaccine than with the qHPV, but always within the range of the background population and none of the congenital anomalies was associated with the vaccination ${ }^{28}$.

\section{SARS-Cov-2 and pregnancy}

CDC data from $29^{\text {th }}$ March 2020 to $14^{\text {th }}$ October reveal that, pregnant women do not have an increased risk of Sars-Cov-2 infection compared to non pregnant women, but the infection of a pregnant woman increases the risk of severe complications ${ }^{30}$. The data regarding the efficiency and safety of COVD19 vaccines in pregnant women are limited, because pregnant women have been excluded from any clinical trial ${ }^{30}$. However vaccination during or shortly before pregnancy do not cause disease but nonspecific side effects from activation of the immune system may occur ${ }^{30,31}$.

\section{Conclusion}

The immune system of women changes and adapts to pregnancy, as is incited by the hormonal and physiological changes developing during pregnancy. The innate immunity becomes enforced and modified in order to prevent the maternal infections. The adaptive immunity seems to be partially suppressed, but the B lymphocytes preserve and enhance their ability to produce immunoglobulins, as is proven by vaccination studies, especially during the $3^{\text {rd }}$ trimester. Fetal immunization is achieved by maternal IgG antibodies transportation through the placenta assisted by neonatal FcRn receptor. There are some proven risks of receiving live attenuated vaccines during pregnancy and for that reason they are contraindicated. However, vaccines containing other ingredients could be given, when the benefits outweigh the potential risks. It is important to stress that, in case of an inadvertent vaccination during 
pregnancy, pregnancy termination should not be recommended.

\section{References}

1. Mor G, Cardenas I, Abrahams V, Guller S. Inflammation and pregnancy: the role of the immune system at the implantation site. Ann N Y Acad Sci. 2011;1221(1):80-87.

2. Kraus, T.A., Engel, S.M., Sperling, R.S. et al. Characterizing the Pregnancy Immune Phenotype: Results of the Viral Immunity and Pregnancy (VIP) Study. J Clin Immunol 32, 2012;300-311.

3. Morelli S, Mandal M, Goldsmith LT, Kashani BN, Ponzio NM. The maternal immune system during pregnancy and its influence on fetal development. Research and Reports in Biology. 2015;6:171-189.

4. Pazos, M., Sperling, R.S., Moran, T.M. et al. The influence of pregnancy on systemic immunity. Immunol Res 54, 2012;254-261.

5. Sperling, Rhoda S. MD; Engel, Stephanie M. PhD; Wallenstein, Sylvan PhD; Kraus, et al. Immunogenicity of Trivalent Inactivated Influenza Vaccination Received During Pregnancy or Postpartum, Obstetrics \& Gynecology: March 2012 - Volume 119 - Issue 3 - p 631-639.

6. Jennifer A. Hutcheon, Deshayne B. Fell, Michael L. Jackson, et al. Detectable Risks in Studies of the Fetal Benefits of Maternal Influenza Vaccination, American Journal of Epidemiology, Volume 184, Issue 3, 1 August 2016, Pages 227-232.

7. Bischoff AL, Følsgaard NV, Carson CG, et al. Altered response to $\mathrm{A}(\mathrm{H} 1 \mathrm{~N} 1)$ pnd09 vaccination in pregnant women: a single blinded randomized controlled trial. PLoS One. 2013 Apr 18;8(4):e56700.

8. Schlaudecker EP, McNeal MM, Dodd CN, Ranz JB, Steinhoff MC. Pregnancy modifies the antibody response to trivalent influenza immunization. J Infect Dis. 2012 Dec 1;206(11):1670-3.

9. Gabriela Vazquez-Benitez, Elyse 0. Kharbanda, Al- lison L. Naleway, et al. Risk of Preterm or Smallfor-Gestational-Age Birth After Influenza Vaccination During Pregnancy: Caveats When Conducting Retrospective Observational Studies, American Journal of Epidemiology, Volume 184, Issue 3, 1 August 2016, Pages 176-186.

10. Sakala IG, Honda-Okubo Y, Fung J, Petrovsky N. Influenza immunization during pregnancy: Benefits for mother and infant. Hum Vaccin Immunother. 2016 Dec;12(12):3065-3071.

11. Andorf S, Bhattacharya S, Gaudilliere B, et al. A pilot study showing a stronger H1N1 influenza vaccination response during pregnancy in women who subsequently deliver preterm. J Reprod Immunol. 2019 Apr;132:16-20.

12. Albrecht M, Arck PC. Vertically Transferred Immunity in Neonates: Mothers, Mechanisms and Mediators. Front Immunol. 2020;11:555. Published 2020 Mar 31. doi:10.3389/fimmu.2020.00555

13. Patricia Palmeira, Camila Quinello, Ana Lúcia Silveira-Lessa, Cláudia Augusta Zago, Magda Carneiro-Sampaio, „IgG Placental Transfer in Healthy and Pathological Pregnancies", Journal of Immunology Research, vol. 2012, Article ID 985646, 13 pages, 2012. https://doi.org/10.1155/2012/985646

14. Rath T, Kuo TT, Baker K, Qiao SW, Kobayashi K, Yoshida M, Roopenian D, Fiebiger E, Lencer WI, Blumberg RS. The immunologic functions of the neonatal Fc receptor for IgG. J Clin Immunol. 2013 Jan;33 Suppl 1(Suppl 1):S9-17. doi: 10.1007/s10875012-9768-y. Epub 2012 Sep 5. PMID: 22948741; PMCID: PMC3548031.

15. Jauniaux E, Jurkovic D, Gulbis B, Liesnard C, Lees C, Campbell S. Materno-fetal immunoglobulin transfer and passive immunity during the first trimester of human pregnancy. Hum Reprod. 1995 Dec;10(12):3297-300. doi: 10.1093/oxfordjournals.humrep.a135906. PMID: 8822462.

16. Weström B, Arévalo Sureda E, Pierzynowska K, Pierzynowski SG, Pérez-Cano FJ. The Immature Gut 
Barrier and Its Importance in Establishing Immunity in Newborn Mammals. Front Immunol. 2020 Jun 9;11:1153. doi: 10.3389/fimmu.2020.01153. PMID: 32582216; PMCID: PMC7296122.

17. Fouda GG, Martinez DR, Swamy GK, Permar SR. The Impact of IgG transplacental transfer on early life immunity. Immunohorizons. 2018;2(1):1425. doi:10.4049/immunohorizons.1700057

18. Helen Y. Chu, Janet A. Englund, Maternal Immunization, Clinical Infectious Diseases, Volume 59, Issue 4, 15 August 2014, Pages 560-568, https:// doi.org/10.1093/cid/ciu327

19. Aaen KH, Anthi AK, Sandlie I, Nilsen J, Mester S, Andersen JT. The neonatal Fc receptor in mucosal immune regulation. Scand J Immunol. 2021 Feb;93(2):e13017. doi: 10.1111/sji.13017. Epub 2021 Jan 17. PMID: 33351196.

20. Psarris A, Sindos M, Daskalakis G, Chondrogianni ME, Panayiotou S, Antsaklis P, Loutradis D. Immunizations during pregnancy: How, when and why. Eur J Obstet Gynecol Reprod Biol. 2019 Sep;240:2935. doi: 10.1016/j.ejogrb.2019.06.019. Epub 2019 Jun 14. PMID: 31226574

21. Swamy GK, Heine RP. Vaccinations for pregnant women. Obstet Gynecol. 2015;125(1)

22. https://www.cdc.gov/vaccines/pregnancy/hcptoolkit/guidelines.html

23. https://www.acog.org/clinical/clinical-guidance/ practice-advisory/articles/2019/04/management-of-pregnant-and-reproductive-aged-women-during-a-measles-outbreak

24. https://obgyn.gr/node/50

25. Keller-Stanislawski B, Englund JA, Kang G, Mangtani P, Neuzil K, Nohynek H, Pless R, Lambach P, Zuber P. Safety of immunization during pregnancy: a review of the evidence of selected in- activated and live attenuated vaccines. Vaccine. 2014 Dec 12;32(52):7057-64. doi: 10.1016/j. vaccine.2014.09.052. Epub 2014 Oct 5. PMID: 25285883

26. McLean HQ, Fiebelkorn AP, Temte JL, Wallace GS; Centers for Disease Control and Prevention. Prevention of measles, rubella, congenital rubella syndrome, and mumps, 2013: summary recommendations of the Advisory Committee on Immunization Practices (ACIP). MMWR Recomm Rep. 2013 Jun 14;62(RR-04):1-34. Erratum in: MMWR Recomm Rep. 2015 Mar 13;64(9):259. PMID: 23760231.

27. Establishment of VARIVAX pregnancy registry. From the Centers for Disease Control and Prevention. JAMA. 1996 Apr 10;275(14):1073. PMID: 8601915.

28. https://www.uptodate.com/contents/immunizations-during-pregnancy (Accessed on March 30, 2021)

29. Faber MT, Duun-Henriksen AK, Dehlendorff C, Tatla MK, Munk C, Kjaer SK. Adverse pregnancy outcomes and infant mortality after quadrivalent HPV vaccination during pregnancy. Vaccine. 2019 Jan 7;37(2):265-271. doi: 10.1016/j. vaccine.2018.11.030. Epub 2018 Nov 28. PMID: 30503078.

30. https://www.uptodate.com/contents/covid19-pregnancy-issues-and-antenatal-care (Accessed on March 30, 2021)

31. https://www.cdc.gov/coronavirus/2019-ncov/ vaccines/recommendations/pregnancy (Accessed on March 30, 2021)

Received 03-03-21

Revised 12-03-21

Accepted 19-03-21 